\title{
Paracoccus homiensis sp. nov., isolated from a sea-sand sample
}

Correspondence

Soon-Wo Kwon

swkwon@rda.go.kr

\author{
Byung-Yong Kim, ${ }^{1}$ Hang-Yeon Weon, ${ }^{2}$ Seung-Hee Yoo, ${ }^{1}$ Soon-Wo Kwon, ${ }^{1}$ \\ Yang-Hee Cho, ${ }^{1}$ Erko Stackebrandt ${ }^{3}$ and Seung-Joo Go ${ }^{1}$ \\ 1,2Korean Agricultural Culture Collection (KACC), Genetic Resources Division, National Institute \\ of Agricultural Biotechnology ${ }^{1}$, and Applied Microbiology Division, National Institute of \\ Agricultural Science and Technology², Rural Development Administration (RDA), \\ Suwon 441-707, South Korea \\ ${ }^{3}$ Deutsche Sammlung von Mikroorganismen und Zellkulturen $\mathrm{GmbH}$, Mascheroder Weg $1 \mathrm{~b}$ \\ D-38124 Braunschweig, Germany
}

\begin{abstract}
Strain DD-R11 ${ }^{\top}$, isolated from a sea-sand sample from Homi Cape, Pohang city, South Korea, was a Gram-negative, aerobic, motile, non-spore-forming, rod- to ovoid-shaped bacterium. Colonies grown on marine agar were circular, convex and colourless to creamy white. Growth occurred between 10 and $40^{\circ} \mathrm{C}$ (optimum $25-30{ }^{\circ} \mathrm{C}$ ) and at $\mathrm{pH} 5 \cdot 0-9 \cdot 0$ (optimum pH 6.0-8.0). The strain could grow in up to $15 \% \mathrm{NaCl}$ (optimum 3-5\% NaCl). According to $16 \mathrm{~S}$ rRNA gene sequence analysis, the strain was a member of the genus Paracoccus in the Alphaproteobacteria. Sequence similarities to type strains of the genus Paracoccus were between $94 \cdot 6$ and $98 \cdot 3 \%$, showing the highest sequence similarity to Paracoccus zeaxanthinifaciens ATCC $21588^{\top}$. The DNA-DNA relatedness value of strain DD-R $11^{\top}$ and $P$. zeaxanthinifaciens ATCC $21588^{\top}$ was $27 \%$. Strain $\mathrm{DD}-\mathrm{R} 11^{\top}$ was characterized by having ubiquinone 10 as the major respiratory quinone and $\mathrm{C}_{18: 1} \omega 7 \mathrm{c}$ as the predominant fatty acid. The DNA G $+\mathrm{C}$ content was $63.0 \mathrm{~mol} \%$. On the basis of its phenotypic and genotypic characteristics, it is suggested that DD-R11 ${ }^{\top}$ represents a novel species of the genus Paracoccus, for which the name Paracoccus homiensis sp. nov. is proposed, with DD-R11 ${ }^{\top}\left(=\operatorname{KACC} 11518^{\top}=\mathrm{DSM} 17862^{\top}\right)$ as the type strain.
\end{abstract}

The genus Paracoccus was proposed by Davis et al. (1969). At the time of writing, the genus contains 19 recognized species with validly published names. Phylogenetic analyses based on 16S rRNA gene sequences have shown that the genus Paracoccus falls within the $\alpha-3$ subgroup of the Proteobacteria. Species of this genus are characterized as Gramnegative and catalase- and oxidase-positive, with a high metabolic versatility and a large amount of $\mathrm{C}_{18: 1} \omega 7 c$ and having ubiquinone 10 as the major isoprenoid quinone.

In this study, a moderately halophilic strain, designated $\mathrm{DD}-\mathrm{R} 11^{\mathrm{T}}$, was characterized on the basis of a polyphasic approach. The strain was isolated from a sea-sand sample collected in Pohang city, Korea. The sample was suspended in a solution of sea salts (Sigma), spread on marine agar 2216 (MA; Difco) and incubated at $28{ }^{\circ} \mathrm{C}$. The isolate was routinely cultured on $\mathrm{MA}$ and maintained as a glycerol suspension $(15 \%, \mathrm{v} / \mathrm{v})$ at $-80{ }^{\circ} \mathrm{C}$.

The isolate was cultivated on MA at $28{ }^{\circ} \mathrm{C}$ for the investigation of morphological and physiological characteristics.

The GenBank/EMBL/DDBJ accession number for the 16S rRNA gene sequence of strain DD-R $11^{\top}$ is DQ342239.
For observation of cell morphology by transmission electron microscopy, cells were grown for $48 \mathrm{~h}$ on MA, negatively stained with $0.5 \%(\mathrm{w} / \mathrm{v})$ uranyl acetate and examined with a LEO model 912AB electron microscope. Tolerance of $\mathrm{pH}$ and temperature was recorded following growth in marine broth at $28{ }^{\circ} \mathrm{C}$ for 21 days. The requirement for $\mathrm{NaCl}$ for growth was tested using nutrient broth supplemented with 0, 1, 3, 5, 7, 10, 15, 20 and 25\% (w/v) NaCl. Phenotypic characteristics such as Gram staining, catalase and oxidase production and hydrolysis of alginic acid, carboxymethylcellulose, casein, gelatin, pectin, tyrosine and starch were performed using the methods of Smibert \& Krieg (1994). Growth under anaerobic conditions was tested in GasPak (BBL) jars at $28{ }^{\circ} \mathrm{C}$ for 21 days. The commercially available API 20NE and API ZYM kits (bioMérieux) were used to determine biochemical properties, utilization of carbohydrates and enzymic activities according to the manufacturer's instructions. Utilization of organic substrates was tested using Biolog GN2 microplates. Colonies suspended in half-strength sea salts (Sigma) solution were used as the inoculum.

The 16S rRNA gene was amplified by PCR using two universal primers as described by Kwon et al. (2003). The 


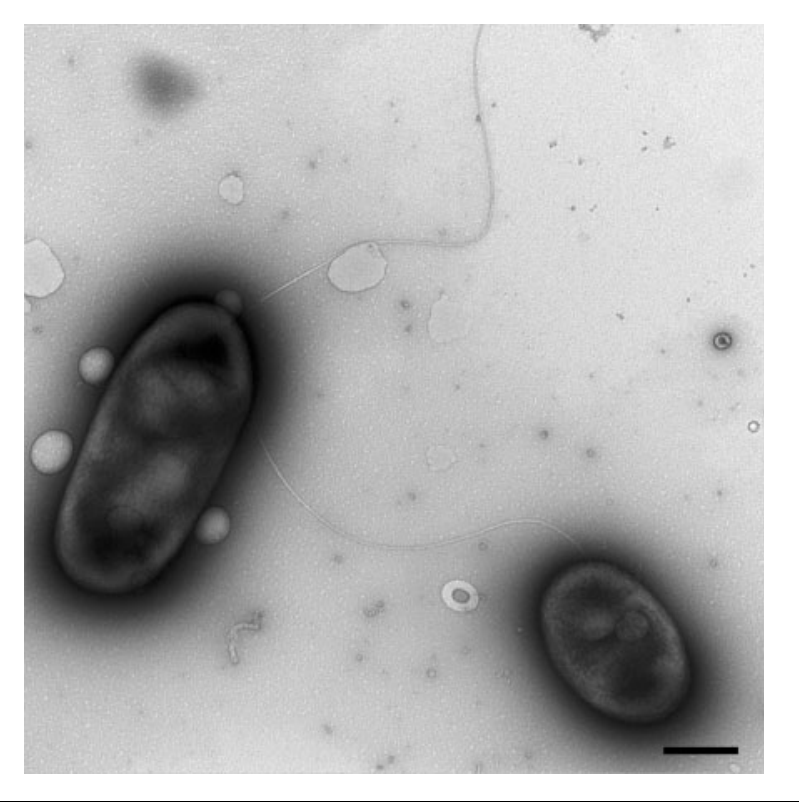

Fig. 1. Transmission electron micrograph of strain DD-R $11^{\top}$. Bar, $500 \mathrm{~nm}$.

sequences of DD-R $11^{\mathrm{T}}$ and related species were aligned using CLUSTAL X (Thompson et al., 1997). The evolutionary distances of 1394 aligned positions were computed using the Kimura two-parameter model (Kimura, 1980) and a phylogenetic tree was generated with MEGA version 2.1 (Kumar et al., 2001) using the neighbour-joining method (Saitou \& Nei, 1987) and evaluated by bootstrap analyses (Felsenstein, 1985) based on 1000 resamplings.

DNA-DNA hybridization was carried out as described by Seldin \& Dubnau (1985). Probe labelling was conducted using the non-radioactive DIG-High Prime system (Roche); hybridized DNA was visualized using the DIG Luminescent Detection kit (Roche). DNA-DNA relatedness was quantified using a densitometer (Bio-Rad). Isoprenoid quinone analysis was performed as described by Groth et al. (1996). The whole-cell fatty acid methyl ester profile was determined using the MIDI Sherlock Microbial Identification system (Microbial ID) with cells of DD-R $11^{\mathrm{T}}$ grown at $28^{\circ} \mathrm{C}$ for $24 \mathrm{~h}$ in MA. The DNA G $+\mathrm{C}$ content was determined according to the method of Mesbah et al. (1989) using a reversed-phase HPLC system with a C18 column.

Cells of strain DD-R $11^{\mathrm{T}}$ were Gram-negative, non-sporeforming, aerobic and catalase- and oxidase-positive. Cells were rod- to ovoid-shaped $(0.7-2.5 \times 0.5-0.7 \mu \mathrm{m})$ and motile with one or more flagella (Fig. 1). They were able to grow on MA. However, they grew weakly on nutrient agar (Difco) and did not grow on trypticase soy agar (Difco) or MacConkey agar (Difco). The strain grew optimally on nutrient broth containing $3-5 \% \mathrm{NaCl}$, grew weakly without $\mathrm{NaCl}$ and did not grow in the presence of more than $15 \%(\mathrm{w} / \mathrm{v}) \mathrm{NaCl}$. After $48 \mathrm{~h}$ on MA, cells formed circular, convex, colourless to creamy white colonies. A phenotypic comparison of DD-R $11^{\mathrm{T}}$ and closely related species of the genus Paracoccus is shown in Table 1.

The major fatty acid for strain DD-R $11^{\mathrm{T}}$ was $\mathrm{C}_{18: 1} \omega 7 c$ $(78 \cdot 9 \%)$. Small amounts of other fatty acids were detected as follows: 11-methyl $\mathrm{C}_{18: 1} \omega 7 c(5 \cdot 8 \%), \mathrm{C}_{18: 0}(4 \cdot 5 \%), \mathrm{C}_{10: 0}$ $3-\mathrm{OH}(2 \cdot 6 \%)$, unidentified ECL $11.799(2 \cdot 4 \%), \mathrm{C}_{12: 0}$ $\operatorname{ALDE}(1 \cdot 8 \%)$ and $\mathrm{C}_{18: 0} 3-\mathrm{OH}(1 \cdot 1 \%)$. This fatty acid profile was similar to those of members of the genus Paracoccus (Kelly et al., 2000) (Table 2).

$16 \mathrm{~S}$ rRNA gene sequencing showed that the sequence of strain DD-R $11^{\mathrm{T}}$ exhibited $94 \cdot 6-98 \cdot 3 \%$ sequence similarity to type strains of the genus Paracoccus. Strain DD-R $11^{\mathrm{T}}$ showed the highest sequence similarity to Paracoccus zeaxanthinifaciens ATCC $21588^{\mathrm{T}}(98 \cdot 3 \%)$, followed by Paracoccus seriniphilus DSM $14827^{\mathrm{T}}(96 \cdot 7 \%)$. The phylogenetic tree showing the position of strain $\mathrm{DD}-\mathrm{R} 11^{\mathrm{T}}$ relative to members of the genus Paracoccus is shown in Fig. 2 . Strain DD-R $11^{\mathrm{T}}$ and P. zeaxanthinifaciens ATCC $21588^{\mathrm{T}}$ formed a compact cluster (100\% bootstrap value), which was loosely related to another cluster including Paracoccus

Table 1. Phenotypic comparison of strain $D D-R 11^{\top}$ with closely related species within the genus Paracoccus

Taxa: 1, DD-R11 ${ }^{\mathrm{T}} ; 2, P$. zeaxanthinifaciens (three strains); 3, P. carotinifaciens IFO $16121^{\mathrm{T}} ; 4, P$. haeundaensis $\mathrm{KCCM} 10460^{\mathrm{T}} ; 5, P$. marcusii DSM $11574^{\mathrm{T}}$; 6, P. seriniphilus DSM $14827^{\mathrm{T}}$. Data from Berry et al. (2003), Pukall et al. (2003), Lee et al. (2004) and this study. +, Positive; -, negative; W, weak; ND, not determined.

\begin{tabular}{|c|c|c|c|c|c|c|}
\hline Characteristic & 1 & 2 & 3 & 4 & 5 & 6 \\
\hline Motility & + & - & + & - & - & - \\
\hline Orange to red pigment & - & + & + & + & + & - \\
\hline Growth at $40{ }^{\circ} \mathrm{C}$ & + & + & - & - & - & - \\
\hline Growth in $6 \% \mathrm{NaCl}$ & + & + & $\mathrm{W}$ & + & $\mathrm{W}$ & + \\
\hline Nitrate reduction & - & - & - & + & - & + \\
\hline Urease & - & + & - & - & - & - \\
\hline \multicolumn{7}{|l|}{ Growth on: } \\
\hline Acetic acid & + & - & $\mathrm{ND}$ & $\mathrm{ND}$ & - & + \\
\hline Adonitol & - & - & + & - & + & + \\
\hline D-Alanine & + & - & $\mathrm{ND}$ & ND & - & + \\
\hline L-Aspartate & - & + & + & $\mathrm{ND}$ & - & + \\
\hline L-Asparagine & + & + & + & - & - & + \\
\hline iso-Erythritol & + & - & + & $\mathrm{ND}$ & + & + \\
\hline Gentiobiose & - & - & + & ND & + & - \\
\hline L-Phenylalanine & + & - & $\mathrm{ND}$ & $\mathrm{ND}$ & - & - \\
\hline Propionic acid & + & - & $\mathrm{ND}$ & $\mathrm{ND}$ & + & + \\
\hline Quinic acid & - & - & + & $\mathrm{ND}$ & + & - \\
\hline L-Rhamnose & + & - & $\mathrm{ND}$ & - & - & - \\
\hline D-Sorbitol & + & - & + & - & + & + \\
\hline Succinic acid & + & - & $\mathrm{ND}$ & $\mathrm{ND}$ & + & - \\
\hline Xylitol & - & - & + & $\mathrm{ND}$ & + & + \\
\hline $\begin{array}{l}\text { DNA G }+ \text { C content } \\
(\mathrm{mol} \%)\end{array}$ & $63 \cdot 0$ & $66 \cdot 9-67 \cdot 7$ & 67 & $66 \cdot 9$ & 66 & $63 \cdot 3$ \\
\hline
\end{tabular}


Table 2. Fatty acid composition of DD-R $11^{\top}$ and its nearest phylogenetic neighbours within the genus Paracoccus

Taxa: 1, DD-R $11^{\mathrm{T}} ; 2, \quad$. zeaxanthinifaciens (mean values from three strains); $3, P$. carotinifaciens IFO $16121^{\mathrm{T}} ; 4, P$. haeundaensis KCCM $10460^{\mathrm{T}}$; 5, P. marcusii DSM $11574^{\mathrm{T}}$; 6, P. seriniphilus DSM $14827^{\mathrm{T}}$. Data from Berry et al. (2003), Pukall et al. (2003), Lee et al. (2004) and this study. Values are percentages of total fatty acids; - , not detected or $<1 \%$.

\begin{tabular}{|lcccccc|}
\hline Fatty acid & $\mathbf{1}$ & $\mathbf{2}$ & $\mathbf{3}$ & $\mathbf{4}$ & $\mathbf{5}$ & $\mathbf{6}$ \\
\hline $\mathrm{C}_{10: 0} 3-\mathrm{OH}$ & $2 \cdot 6$ & $4 \cdot 9$ & $3 \cdot 4$ & $2 \cdot 1$ & $6 \cdot 2$ & $7 \cdot 9$ \\
Unidentified ECL $11 \cdot 799$ & $2 \cdot 4$ & $3 \cdot 6$ & $2 \cdot 8$ & - & $4 \cdot 9$ & - \\
Unidentified ECL $15 \cdot 275$ & - & $1 \cdot 6$ & $1 \cdot 1$ & - & $2 \cdot 9$ & - \\
$\mathrm{C}_{12: 1} \omega 7 c$ & - & - & - & $2 \cdot 0$ & - & - \\
$\mathrm{C}_{12: 0}$ ALDE & $1 \cdot 8$ & - & - & - & - & - \\
$\mathrm{C}_{14: 0} 3-\mathrm{OH}$ & - & - & - & $1 \cdot 5$ & - & - \\
$\mathrm{C}_{18: 1} \omega 7 c$ & $78 \cdot 9$ & $80 \cdot 2$ & $84 \cdot 0$ & $84 \cdot 3$ & $80 \cdot 3$ & $83 \cdot 5$ \\
$\mathrm{C}_{18: 0}$ & $4 \cdot 5$ & $3 \cdot 6$ & $5 \cdot 2$ & $7 \cdot 8$ & $2 \cdot 6$ & $2 \cdot 5$ \\
$\mathrm{C}_{18: 0} 3-\mathrm{OH}$ & $1 \cdot 1$ & - & - & - & - & - \\
$11-\mathrm{Methyl} \mathrm{C} \mathrm{C}_{18: 1} \omega 7 c$ & $5 \cdot 8$ & - & - & - & - & $1 \cdot 5$ \\
Summed feature $2 *$ & - & $2 \cdot 9$ & $2 \cdot 1$ & - & $3 \cdot 0$ & - \\
\hline
\end{tabular}

${ }^{*}$ Summed feature 2 comprises $\mathrm{C}_{14: 0} 3-\mathrm{OH}$ and/or $\mathrm{C}_{16: 1}$ iso I.

seriniphilus DSM $14827^{\mathrm{T}}$, Paracoccus carotinifaciens IFO $16121^{\mathrm{T}}$, Paracoccus marcusii DSM $11574^{\mathrm{T}}$ and Paracoccus haeundaensis KCCM $10460^{\mathrm{T}}$.

According to DNA-DNA hybridization, strain DD-R $11^{\mathrm{T}}$ showed a DNA-DNA relatedness value of $27 \%$ to $P$. zeaxanthinifaciens ATCC $21588^{\mathrm{T}}$. The levels obtained for $16 \mathrm{~S}$ rRNA gene similarity $(<97 \%)$ and DNA-DNA hybridization $(<70 \%)$ support the genomic distinction of strain DD-R $11^{\mathrm{T}}$ from other species in the genus Paracoccus (Stackebrandt \& Goebel, 1994; Wayne et al., 1987). On the basis of the phylogenetic, chemotaxonomic and phenotypic data, strain DD-R $11^{\mathrm{T}}$ should be classified as the type strain of a novel species of the genus Paracoccus, for which we propose the name Paracoccus homiensis sp. nov.
Description of Paracoccus homiensis sp. nov.

Paracoccus homiensis (ho.mi.en'sis. N.L. masc. adj. homiensis pertaining to Homi Cape, South Korea, where the type strain was isolated).

Cells are Gram-negative, aerobic, motile, non-spore-forming and rod- to ovoid-shaped $(0.7-2.5 \times 0.5-0.7 \mu \mathrm{m})$. Colonies on MA are circular, convex and colourless to creamy white. Growth occurs between 10 and $40{ }^{\circ} \mathrm{C}$ (optimum $25-30{ }^{\circ} \mathrm{C}$ ) and at $\mathrm{pH} 5 \cdot 0-9 \cdot 0$ (optimum pH $6 \cdot 0-8 \cdot 0)$. Grows optimally at $3-5 \% \mathrm{NaCl}$ and weakly at $15 \%(\mathrm{w} / \mathrm{v}) \mathrm{NaCl}$. Positive for catalase, oxidase and hydrolysis of aesculin, gelatin, Tween 80 and tyrosine. Negative for nitrate reduction, indole production, glucose fermentation, arginine dihydrolase, hydrolysis of alginic acid, casein, carboxymethylcellulose, pectin, starch and urea. Enzymic activity is observed for alkaline phosphatase, esterase (C4), esterase lipase (C8), leucine arylamidase, cystine arylamidase, acid phosphatase, naphthol-AS-BIphosphohydrolase, $\alpha$-galactosidase, $\beta$-galactosidase and $\alpha$-glucosidase, but no activity is observed for lipase (C14), valine arylamidase, trypsin, $\alpha$-chymotrypsin, $\beta$-glucuronidase, $\beta$-glucosidase, $N$-acetyl- $\beta$-glucosaminidase, $\alpha$-mannosidase and $\alpha$-fucosidase. Utilizes Tween 80 , D-arabitol, D-cellobiose, iso-erythritol, D-fructose, D-galactose, $\alpha$-Dglucose, myo-inositol, $\alpha$-D-lactose, D-mannitol, D-mannose, D-melibiose, L-rhamnose, D-sorbitol, sucrose, D-trehalose, turanose, pyruvic acid methyl ester, succinic acid monomethyl ester, acetic acid, cis-aconitic acid, citric acid, formic acid, D-gluconic acid, D-glucuronic acid, $\beta$-hydroxybutyric acid, $p$-hydroxyphenylacetic acid, DL-lactic acid, propionic acid, D-saccharic acid, succinic acid, D-alanine, L-alanine, L-asparagine, L-aspartic acid, L-glutamic acid, L-leucine, L-ornithine, L-phenylalanine, L-proline, L-serine, phenylethylamine and glycerol. Does not utilize $\alpha$-cyclodextrin, dextrin, glycogen, Tween 40, $N$-acetyl-D-galactosamine, $N$-acetyl-D-glucosamine, adonitol, L-arabinose, L-fucose, gentiobiose, lactulose, maltose, methyl $\beta$-D-glucoside, D-psicose, xylitol, D-galactonic acid lactone, D-galacturonic acid, D-glucosaminic acid, $\alpha$-hydroxybutyric acid,

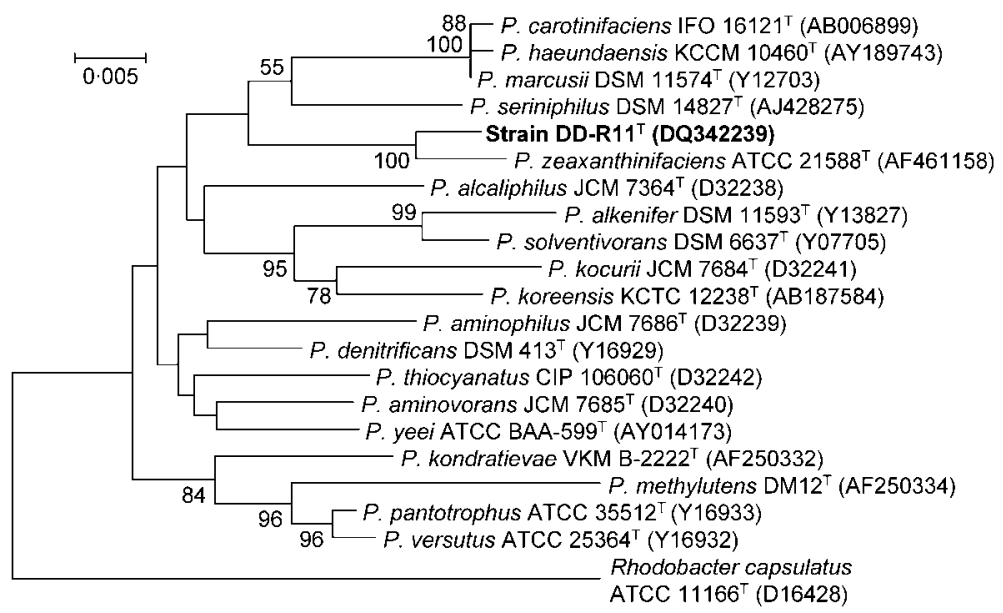

http://ijs.sgmjournals.org
Fig. 2. Neighbour-joining tree showing the relationship of strain DD-R $11^{\top}$ to other members of the genus Paracoccus based on 16S rRNA gene sequences. Only bootstrap percentages above $50 \%$ are shown (1000 replications). The sequence of Rhodobacter capsulatus ATCC $11166^{\top}$ was used as the outgroup. Bar, $0.5 \%$ sequence divergence. 
$\gamma$-hydroxybutyric acid, itaconic acid, $\alpha$-ketobutyric acid, $\alpha$-ketoglutaric acid, $\alpha$-ketovaleric acid, malonic acid, quinic acid, sebacic acid, bromosuccinic acid, succinamic acid, glucuronamide, L-alaninamide, L-alanyl glycine, glycyl L-aspartic acid, glycyl L-glutamic acid, L-histidine, hydroxy-L-proline, L-pyroglutamic acid, D-serine, L-threonine, DL-carnitine, $\gamma$-aminobutyric acid, urocanic acid, inosine, uridine, thymidine, putrescine, 2-aminoethanol, 2,3-butanediol, DL- $\alpha$-glycerol phosphate, $\alpha$-D-glucose 1-phosphate and D-glucose 6-phosphate. Ubiquinone Q-10 is the major isoprenoid quinone. The major fatty acid is $\mathrm{C}_{18: 1} \omega 7 \mathrm{c}$. The $\mathrm{G}+\mathrm{C}$ content is $63.0 \mathrm{~mol} \%$.

The type strain, DD-R11 ${ }^{\mathrm{T}} \quad\left(=\mathrm{KACC} \quad 11518^{\mathrm{T}}=\mathrm{DSM}\right.$ $17862^{\mathrm{T}}$ ), was isolated from a sea-sand sample collected from Homi Cape, Pohang city, South Korea.

\section{Acknowledgements}

This work was supported by a grant (203068-03-2-SB010) from the Agricultural R\&D Promotion Center, South Korea.

\section{References}

Berry, A., Janssens, D., Hümbelin, M. \& 10 other authors (2003). Paracoccus zeaxanthinifaciens sp. nov., a zeaxanthin-producing bacterium. Int J Syst Evol Microbiol 53, 231-238.

Davis, D. H., Doudoroff, M., Stanier, R. Y. \& Mandel, M. (1969). Proposal to reject the genus Hydrogenomonas: taxonomic implications. Int J Syst Bacteriol 19, 375-390.

Felsenstein, J. (1985). Confidence limits on phylogenies: an approach using the bootstrap. Evolution 39, 783-791.

Groth, I., Schumann, P., Weiss, N., Martin, K. \& Rainey, F. A. (1996). Agrococcus jenensis gen. nov., sp. nov., a new genus of actinomycetes with diaminobutyric acid in the cell wall. Int J Syst Bacteriol 46, 234-239.

Kelly, D. P., Rainey, F. A. \& Wood, A. P. (2000). The genus Paracoccus. In The Prokaryotes: an Evolving Electronic Resource for the Microbiological Community, 3rd edn. Edited by M. Dworkin, N. Falkow, H. Rosenberg, K.-H. Schleifer \& E. Stackebrandt. New York: Springer-Verlag.
Kimura, M. (1980). A simple method for estimating evolutionary rates of base substitutions through comparative studies of nucleotide sequences. J Mol Evol 16, 111-120.

Kumar, S., Tamura, K., Jakobsen, I.-B. \& Nei, M. (2001). MEGA: molecular evolutionary genetics analysis software. Bioinformatics 17, 1244-1245.

Kwon, S. W., Kim, J. S., Park, I. C., Yoon, S. H., Park, D. H., Lim, C. K. \& Go, S. J. (2003). Pseudomonas koreensis sp. nov., Pseudomonas umsongensis sp. nov. and Pseudomonas jinjuensis sp. nov., novel species from farm soils in Korea. Int J Syst Evol Microbiol 53, 21-27.

Lee, J. H., Kim, Y. S., Choi, T.-J., Lee, W. J. \& Kim, Y. T. (2004). Paracoccus haeundaensis sp. nov., a Gram-negative, halophilic, astaxanthinproducing bacterium. Int J Syst Evol Microbiol 54, 1699-1702.

Mesbah, M., Premachandran, U. \& Whitman, W. B. (1989). Precise measurement of the $\mathrm{G}+\mathrm{C}$ content of deoxyribonucleic acid by highperformance liquid chromatography. Int J Syst Bacteriol 39, 159-167.

Pukall, R., Laroche, M., Kroppenstedt, R. M., Schumann, P., Stackebrandt, E. \& Ulber, R. (2003). Paracoccus seriniphilus sp. nov., an L-serine-dehydratase-producing coccus isolated from the marine bryozoan Bugula plumosa. Int J Syst Evol Microbiol 53, 443-447.

Saitou, N. \& Nei, M. (1987). The neighbor-joining method: a new method for reconstructing phylogenetic trees. Mol Biol Evol 4, 406-425.

Seldin, L. \& Dubnau, D. (1985). Deoxyribonucleic acid homology among Bacillus polymyxa, Bacillus macerans, Bacillus azotofixans, and other nitrogen-fixing Bacillus strains. Int J Syst Bacteriol 35, 151-154.

Smibert, R. M. \& Krieg, N. R. (1994). Phenotypic characterization. In Methods for General and Molecular Bacteriology, pp. 607-654. Edited by P. Gerhardt, R. G. E. Murray, W. A. Wood \& N. R. Krieg. Washington, DC: American Society for Microbiology.

Stackebrandt, E. \& Goebel, B. M. (1994). Taxonomic note: a place for DNA-DNA reassociation and 16S rRNA sequence analysis in the present species definition in bacteriology. Int J Syst Bacteriol 44, 846-849.

Thompson, J. D., Gibson, T. J., Plewniak, F., Jeanmougin, F. \& Higgins, D. G. (1997). The CLUSTAL_X windows interface: flexible strategies for multiple sequence alignment aided by quality analysis tools. Nucleic Acids Res 25, 4876-4882.

Wayne, L. G., Brenner, D. J., Colwell, R. R. \& 9 other authors (1987). International Committee on Systematic Bacteriology. Report of the ad hoc committee on reconciliation of approaches to bacterial systematics. Int J Syst Bacteriol 37, 463-464. 